\title{
Timing Synchronization Algorithm for OFDM-UWB Systems Based on IEEE 802.15.3c
}

\author{
Chunyu Li, Shuqian He, Zhengjie Deng, Caixia Chen and Chun Shi* \\ School of Information Science and Technology, Hainan Normal University, Haikou, China \\ ${ }^{*}$ Corresponding author
}

\begin{abstract}
According to the $60 \mathrm{GHz}$ OFDM-UWB system of IEEE 802.15.3c standard, the characteristics of the preamble Golay complementary sequence are analyzed for better timing synchronization scheme that can resist multipath interference. The normalized autocorrelation method is used to detect the falling edge for frame detection and coarse timing. And then, the autocorrelation of the Gray complementary sequence is used to find the average value of several peaks for fine timing. Simulation results show that the proposed scheme can control the residual timing offset, and the algorithm has low complexity and high timing accuracy. Moreover, the MSRE with fine timing can reach 10-3 orders of magnitude in the CM1 multipath channel with low SNR, which effectively resists multipath interference.
\end{abstract}

Keywords_IEEE 802.15.3c proposal; golay complementary sequences; synchronization; anti-multipath interference

\section{INTRODUCTION}

With the development of wireless communication and the demand for high-speed wireless transmission rate, two standards of the IEEE 802.15.3a and ECMA-368 support Orthogonal Frequency Division Multiplexing Ultra Wide Band (OFDM-UWB) system with the maximum transmission rate of Mbit/s level, which obviously has been unable to meet needs of bandwidth. The IEEE 802.15.3c standard [1] is available for Wireless Personal Area Network (WPAN), which operates primarily in the $60 \mathrm{GHz}$ radio band with data rates exceeding Gbps. In which, the OFDM is one of the modulation schemes due to its high spectral efficiency, strong anti-inter-symbol interference (ISI) and strong anti-frequency selective fading ability. To meet such a high data rate, the OFDM system uses higher orthogonal carriers. If the timing is advanced and does not exceed the length of the cyclic prefix, this will bring frequency phase rotation and inter-carrier interference (ICI), which requires channel estimation for compensation. If the timing is delayed, this will cause very serious inter-symbol interference (ISI), the system performance will decrease [2].

For the synchronization problem, a lot of synchronization algorithms have been proposed. In the maximum likelihood (ML) method [3], the estimation accuracy is high, and it needs another channel estimation algorithm to estimate the signal-to -noise ratio (SNR); The performance is better than the ML method in the maximum posteriori probability (MAP) method [4], but it needs to know the channel parameters; The method of maximum autocorrelation method based on the synchronization sequence [5] does not need channel estimation, but it is sensitive to multipath. These algorithms cannot reduce multipath interference effectively.

\section{GOLAy COMPLEMENTARY SEQUENCES}

The Golay complementary sequences are defined as a pair of equal length sequences, which consists of two kinds of elements. The Golay complementary sequences have the property that the logarithm of the same element in sequences number of one pairs is equal to that of another pairs with the same separation [6]. The Golay complementary sequences have autocorrelation properties.

Let $a(n)=\left(\alpha_{1}, \alpha_{2}, \ldots, \alpha_{N}\right), b(n)=\left(\beta_{1}, \beta_{2}, \ldots, \beta_{N}\right)$ be sequences of length $N\left(a_{n} \in\{+1,-1\}, b_{n} \in\{+1,-1\}\right)$. The respective autocorrelation functions are defined as:

$$
\begin{aligned}
& R_{b}(j)=\sum_{i=0}^{N-j-1} \beta_{i} \beta_{i+j} \\
& R_{a}(j)=\sum_{i=0}^{N-j-1} \alpha_{i} \alpha_{i+j}
\end{aligned}
$$

The sum of the correlation functions is:

$$
\begin{gathered}
R_{a}(j)+R_{b}(j)=0 \quad j \neq 0 \\
R_{a}(0)+R_{b}(0)=2 N
\end{gathered}
$$

where a pair of sequences $a(n)$ and $b(n)$ is Golay complementary sequences. The Golay complementary sequences have a simple structure without any requirement for length, and there is no limitation on the channel delay extension. Moreover, due to the complementary autocorrelation characteristics of the sequence, the non-zero output will be obtained only when the sequences are completely correlated, and the output is the channel response. Therefore, Golay complementary sequence can resist multipath interference. The principle of using Golay complementary sequence for channel response estimation is that if the channel response is $h(n)$, the Golay complementary sequence is transmitted as described above, then the convolution of the transmit sequence and the channel response are:

$$
r_{a}(n)=a(n) * h(n)
$$




$$
r_{b}(n)=b(n) * h(n)
$$

By calculating the correlation value with the local sequence respectively at the receiving end, we have:

$$
\begin{aligned}
R(n) & =r_{a}(n) * a(-n)+r_{b}(n) * b(-n) \\
& =[a(n) * a(-n)+b(n) * b(-n)] * h(n) \\
& =\left[R_{a}(n)+R_{b}(n)\right] * h(n) \\
& =V h(n)
\end{aligned}
$$

where $V$ is a constant.

A general synthesis of complementary sequences is to form higher order complementary sequences from lower order complementary sequences. A sequence of $b$ is connected to another sequence of $a$ to form a high order sequence; and then, $\bar{b}$ is connected to the other end of $a$ to form another high order sequence, which forms a pair of complementary sequences.
Sequence $\bar{b}$ is to replace all elements in sequence $b$ with another element [7]. The preamble sequences of $a_{128}, b_{128}, a_{256}$ and $b_{256}$ defined in the IEEE802.15.3c standard are the Golay complementary sequences, i.e., $a_{256}$ and $b_{256}$ can be represented by the following formula:

$$
\begin{aligned}
& a_{256}=\left[\begin{array}{ll}
a_{128} & b_{128}
\end{array}\right] \\
& b_{256}=\left[\begin{array}{ll}
a_{128} & \bar{b}_{128}
\end{array}\right]
\end{aligned}
$$

\section{SYNCHRONIZATION ALGORITHM}

IEEE 802.15.3c standard specifies the system of the two frame structure, in which the first frame structure is used in this paper. As shown in Figure I, the frame structure is composed of preamble, header and payload. The preamble consists of frame synchronization (SYNC) sequence, start frame delimiter (SFD) and channel estimation sequence (CES). The SYNC contains a repetitive sequence to achieve higher robustness.

The Golay complementary sequence has excellent autocorrelation characteristics, no side lobe and simple structure. It has no limitation on the delay expansion length of the channel, and can resist multi-path interference. We can use these characteristics for synchronous capture.

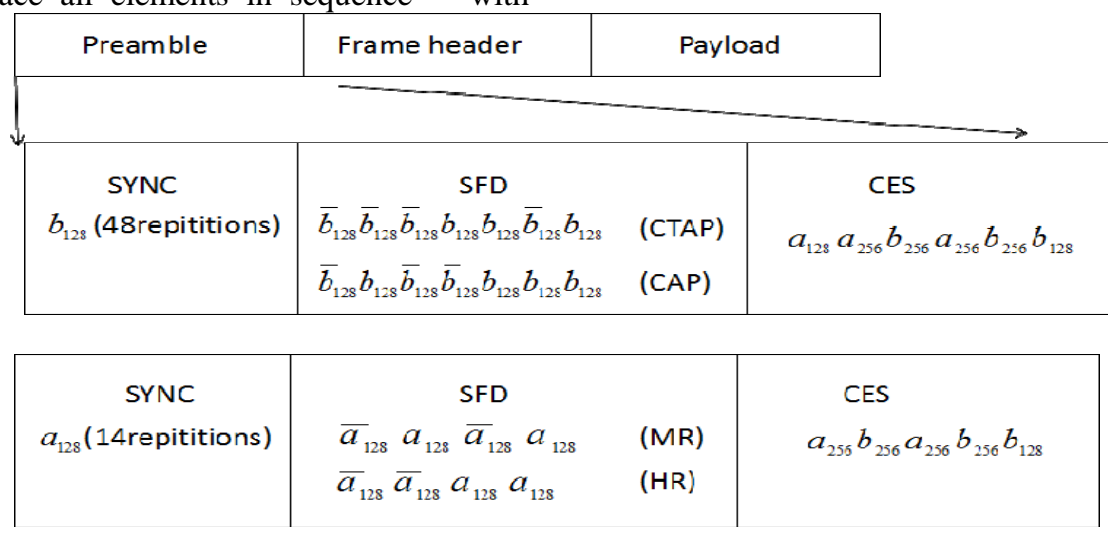

FIGURE I. EXAMPLE OF A ONE-COLUMN FIGURE CAPTION

\section{COARSE SYMBOL SYNCHRONIZATION}

Rough timing synchronization means a rough symbol timing synchronization. IEEE802.15.3c provides that the first part of the synchronization sequence SYNC is designed as a duplicate structure, each of which is a Golay sequence of 128 . The key to detecting the preamble is to set the threshold reasonably. However, in the signal transmission of the path of sight and non-sight distance, the amplitude of the signal is distinctly different. Therefore, the amplitude of the threshold is very difficult to determine. The threshold is too high, which may lead to missed detection in NLOS path detection, and a false alarm is formed. However, if the threshold is too low, it may lead to noise interference, false alarm and false alarm. Therefore, in order to facilitate the definition of threshold value, the normalized two-dimensional sliding correlation can be used, and the normalized high level value is 1 .

$$
x(n)=\left|\frac{\sum_{i=0}^{127} p^{*}(n+i) \times p(n+i+128)}{\sum_{i=0}^{127} p^{*}(n+i) \times p(n+i)}\right|
$$

where $p(n)$ is the received signal whose window length is 128 and a threshold $\mathrm{G}$ is determined by simulation. When two consecutive $x(n)$ are changed from greater than $G$ to less than $\mathrm{G}$, a falling edge is detected, which means that the frame is detected. Since the first part of the preamble SYNC is a known repetitive structure, the length is $I$, as shown in Eqn. (6), we can see that this part will produce a flat peak, and the second part of the preamble is SFD, which is different from SYNC. At the 
junction of these two parts, it will produce a falling edge. Let $n=u$, we can define the coarse timing position is $\mathrm{d}$.

$$
d=I-u
$$

\section{FINE SYMBOL SYNCHRONIZATION}

In the fine symbol synchronization phase, we can correct the smaller synchronization deviation at coarse symbol synchronization and track the deviation in synchronization within one frame. In order to reduce the complexity of the algorithm, the length of the window is reduced to 32, which means that the $b_{128}$ of the synchronous SYNC sequence can be separated by the following equation.

$$
b_{128}=\left[\begin{array}{llll}
a_{32} & b_{32} & \bar{a}_{32} & b_{32}
\end{array}\right]
$$

The sequences $a_{32}$ and $b_{32}$ maintain the characteristic of Golay complementary. The two windows with two lengths 32 bits are taken to correlate and normalize like the normalized maximum correlation (NMC) operation.

$$
x(n)=\frac{\left|\sum_{k=0}^{31} p(n+k) \times p(n+k+64)^{*}+\sum_{k=0}^{31} p(n+k) \times p(n+k+128)^{*}\right|}{\left|\sum_{k=0}^{31} p^{*}(n+k) \times p(n+k)\right|}
$$

If the current window is $a_{32}$ and $\bar{a}_{32}$, the normalized ideal correlation value is -1 . If the current window is $b_{32}$ and $\bar{b}_{32}$, the normalized ideal correlation value is +1 . And if the absolute value operation is performed, there is a zero value between the peaks of the correlation value. Because of its zero crossing characteristic, a very low threshold can be defined. When the threshold value $x(n)$ is greater than this threshold, the peak value of every 64 samples in the frame synchronization sequence (SYNC) is $k$ with $m$ peaks. Average the M peak to the last peak and mark it as a symbol $L$ for the fine timing. As shown in the following formula:

$$
L=\sum_{n=1}^{m}[k(n)+(m-n) * 64] / m
$$

Through the simulation, the number of the correlation peaks in the frame synchronization sequence (SYNC) is certain. As long as these peaks are found, the error produced in the rough timing stage will not affect the precision of the fine timing. And because of the complementary characteristics of the Golay sequence, the detected indexes are not affected by the multipath delay, so the algorithm has a high accuracy.

\section{Algorithm Performance SimUlation}

In order to verify the proposed synchronization scheme, the HSI (MCS7) model [1] based on the IEEE802.15.3c standard is used to build the simulation platform. The first is the $5 / 8$ channel encoding rate, then using 64QAM modulation signal mapping, followed by the OFDM sub module to modulate and transmit. OFDM has 512 sub carrier sub module, 336 of which are modulated by sending data, 16 pilot symbols inserted, the remaining 160 is zero form virtual sub carrier, by 512 points and finish IFFT modulation. The length of the cyclic prefix added is $1 / 8$ of the length of the data symbol, that is, 64 sample values, so the number of sampling points for a complete OFDM symbol is 576. The symbol period is about $218.18 \mathrm{~ns}$, and the bandwidth of the system is about $2640 \mathrm{MHz}$. The additive white Gauss noise channel (AWGN) is used in the general channel, and the multipath channel selects CM1 in the $60 \mathrm{GHz}$ channel of the Tg3c group and the CM2 channel model [8]. The OFDM receiver module structure is similar to the sending sub module, but it is only its inverse operation, and it is no longer repeated here. Different thresholds corresponding to the false alarm and missed alarm probability are different. From the Figure 2, if the signal to noise ratio is $0 \mathrm{~dB}$ in the CM1, CM2 channel with the threshold between 0.14 and 0.16 , the frame detection is accurate and we take 0.15 as the coarse decision threshold.

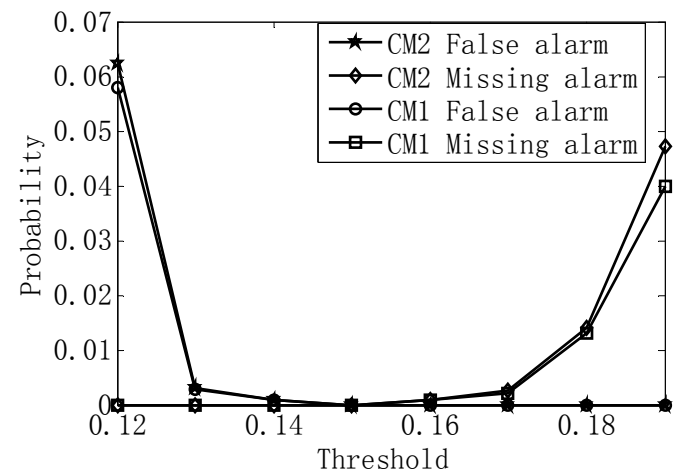

FIGURE II. DIFFERENT THRESHOLD CORRESPONDING TO THE PROBABILITY OF FALSE ALARM AND MISSED ALARM

The OFDM UWB system specified by IEEE802.15.3c is simulated, and the RMS error (MSRE) curve of coarse and fine timing deviation in Gauss and multipath channels is simulated respectively. Figure III is the MSRE with coarse timing under different channels. It can be seen that under the Gauss channel and the multipath channel CM1, the MSRE of coarse timing is not very different, but under the CM2 channel, the coarse timing MSRE is relatively large. With the increase of SNR, MSRE is stable at 5 sample values. Because just use the coarse timing phase repeats is two-dimensional sliding relevant results, and did not use complementary Golay sequences, so the anti-multipath performance is not ideal, in low SNR, the coarse timing of MSRE is relatively large, so still need to further reduce the fine timing deviation. Figure IV is a MSRE of fine timing under different channels. Under the Gauss and CM1 channels of $14 \mathrm{~dB}$, MSRE can reach 10-3 orders of magnitude and MSRE of CM2 can reach 10-2 orders of magnitude. Fine timing uses the complementary correlation of sequences to normalize the separation and the maximum correlation operation. After adding the related results, the timing accuracy is improved, and the timing deviation can be controlled in a smaller range. It can be seen that the algorithm can resist multipath interference and can make accurate timing. 


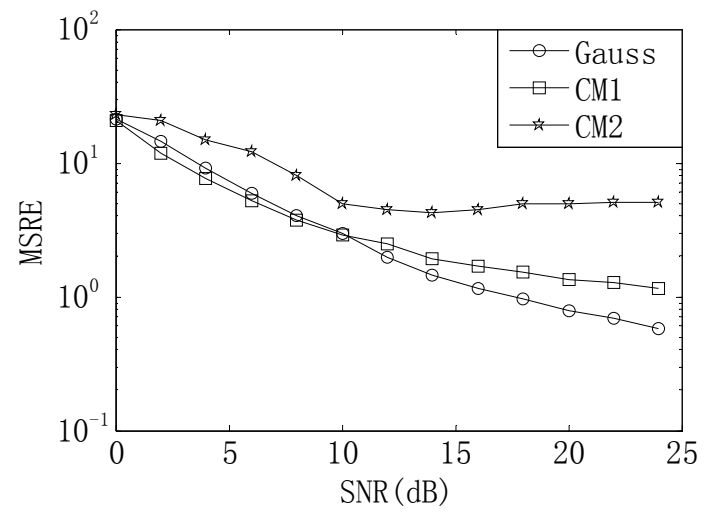

FIGURE III. THE MSRE OF COARSE TIMING

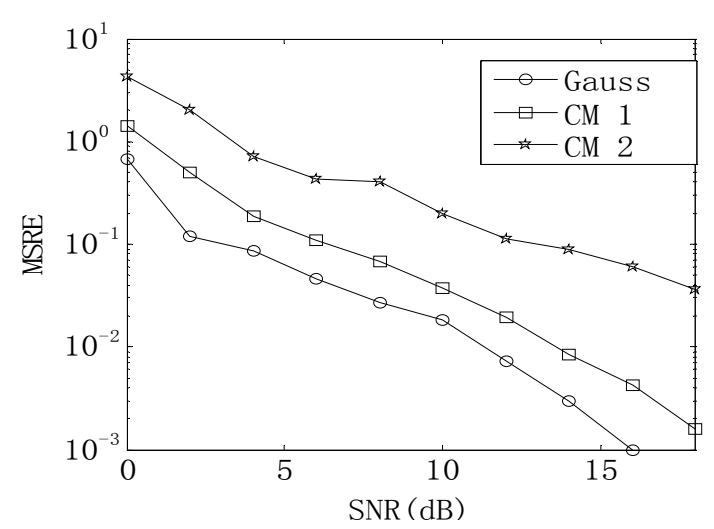

FIGURE IV.THE MSRE OF FINE TIMING

\section{CONCLUSION}

For the OFDM ultra wideband (UWB) system of IEEE 802.15.3c, a complete timing synchronization scheme for the system is given through algorithm simulation. Using the Golay complementary sequence to resist the multipath, the effective correction of the timing deviation is achieved in the multipath channel with a low complexity algorithm. The scheme takes into account the timing synchronization performance and the complexity of the system implementation.

\section{ACKNOWLEDGEMENT}

This research was financially supported by the National Natural Science Foundation (61362016, 61562023, 61502127), Colleges and Universities Scientific Research Projects of Hainan Province (Hnky2016-17, HNJG2014-33), Hainan Province Natural Science Fund (617121, 617110), National special program of international scientific and technological cooperation(2014DFA13140).

\section{REFERENCES}

[1] IEEE. Wireless medium access control (MAC) and physical layer (PHY) specifications for high rate wireless personal area networks (WPAN). IEEE Std. P802.15.3c[S]. Piscataway, NJ: IEEE Standards Association, 2009.

[2] Chen M, He J, Cao Z, et al. Symbol synchronization and sampling frequency synchronization techniques in real-time DDO-OFDM systems[J]. Optics Communications, 2014, 326(5):80-87.
[3] Minn, H, Bhargava, V.K, Letaief, K.B. A combined timing and frequency synchronization and channel estimation for OFDM[J]. IEEE Transactions on Communications, 2006, 54(3):416-422.

[4] Berger C R, Zhou S, Tian Z, et al. Performance analysis on an MAP fine timing algorithm in UWB multiband OFDM[J]. IEEE Transactions on Communications, 2008, 56(10):1606-1611.

[5] Li Y, Minn H, Rajatheva R M A. Synchronization, Channel Estimation, and Equalization in MB-OFDM Systems[M]. IEEE Press, 2008.

[6] Yao Z, Guanglong L I, Wang S, et al. Compressed Sensing Channel Estimation Algorithm Based on Deterministic Sensing with Golay Complementary Sequences[J]. Journal of Electronics \& Information Technology, 2016.

[7] Wu Z. Application of Golay Complementary Series to Spectral Analysis[J]. Journal of Electronic Measurement and Instrument1996, 10(3): $14-18$

[8] Zhang $\mathrm{H}, \mathrm{Xu} \mathrm{N}$, Wang J, et al. On the capacity of $60 \mathrm{GHz}$ wireless communications over IEEE 802.15.3c channel models[C].IEEE Pacific Rim Conference on Communications, Computers and Signal Processing (PacRim),Victoria, BC ,Canada,Aug.23- 26, 2011 ,680-684. 